K. Matsumoto

Nagoya Math. J.

Vol. 116 (1989), 123-138

\title{
A PROBABILISTIC STUDY ON THE VALUE-DISTRIBUTION OF DIRICHLET SERIES ATTACHED TO CERTAIN CUSP FORMS
}

\author{
KOHJI MATSUMOTO
}

\section{§1. Introduction}

The existence of the asymptotic probability measure of the Riemann zeta-function was proved in Bohr-Jessen's classical paper [3] [4].

Let $s=\sigma+i t$ be a complex variable, $\zeta(s)$ the Riemann zeta-function, and $R$ an arbitrary rectangle with the edges parallel to the axes. Then, for any $\sigma_{0}>1 / 2$ and $T>0$, the set

$$
\left\{t \in[0, T] \mid \log \zeta\left(\sigma_{0}+i t\right) \in R\right\}
$$

is Jordan measurable, and we denote the Jordan measure of this set by $V(T, R ; \zeta)$. Then, Bohr-Jessen's main result asserts the existence of the limit

$$
W(R ; \zeta)=\lim _{T \rightarrow \infty} V(T, R ; \zeta) / T,
$$

which we call the asymptotic probability measure of $\log \zeta(s)$ on the line $\sigma=\sigma_{0}$.

Let $N$ be a positive integer, $\theta_{n} \in[0,1)(1 \leqq n \leqq N)$, and we define the mapping $S_{N}$ from $Q_{N}=[0,1)^{N}$ to the complex plane $C$ by

$$
S_{N}\left(\theta_{1}, \cdots, \theta_{N} ; \zeta\right)=-\sum_{n=1}^{N} \log \left(1-p_{n}^{-\sigma_{0}} \exp \left(2 \pi i \theta_{n}\right)\right),
$$

where $p_{n}$ is the $n$-th prime number. By $W_{N}(R ; \zeta)$ we mean the $N$-dimensional Jordan measure of the inverse image $S_{N}^{-1}(R)$. Then, Bohr-Jessen proved that when $N$ tends to infinity, the $\operatorname{limit} \lim W_{N}(R ; \zeta)$ exists, which just coincides our desired $W(R ; \zeta)$.

Here we take notice of the property that in the right-hand side of (1.1), each term $\log \left(1-p_{n}^{-\sigma_{0}} e^{2 \pi i \theta_{n}}\right)$ describes a closed convex curve, as $\theta_{n}$

Received June 29, 1988. 
moves from 0 to 1 . Hence, $S_{N}\left(\theta_{1}, \cdots, \theta_{N}\right)$ is a kind of "sum" of convex curves. Bohr-Jessen's original proof of the existence of $\lim W_{N}(R ; \zeta)$ is based on a rather involved theory on the infinite sums of convex curves [5]. Later, using Fourier transforms of probability measures, an alternative proof was given ([6] [13]), but it also treats the case of convex curves only (see Theorem 13 of [13]).

For more general Euler products, however, the corresponding terms do not always describe convex curves any more. Therefore, if we want to generalize Bohr-Jessen's theory, it is indispensable to develop a method which is independent of convexity. In the present paper, we will study the value-distribution of Dirichlet series attached to cusp forms which are simultaneous eigenfunctions of Hecke operators, as a simple example of non-convex Euler products.

In the following sections, the rectangles we consider are closed and have the edges parallel to the axes. For any $z \in C$ and subset $X \subset C$, the set $\{w-z \mid w \in X\}$ we denote by $X-z$. Also, dist $(z, X)$ means the lower bound of $\{|z-w| \mid w \in X\}$.

\section{§2. Statement of results}

As usual, we denote by $\mathrm{SL}(2, Z)$ the elliptic modular group. Let $k$, $M$ be posititive integers, $\chi$ a Dirichlet character mod. $M$, and we define the Hecke congruence subgroup of level $M$ by

$$
\Gamma_{0}(M)=\left\{\left[\begin{array}{ll}
a & b \\
c & d
\end{array}\right] \in \mathrm{SL}(2, Z) \mid c \equiv 0(\bmod . M)\right\}
$$

By $\mathscr{S}_{k}(M, \chi)$ we mean the space of cusp forms of weight $k$ with respect to $\Gamma_{0}(M)$ with character $\chi$. If a function $f(w)$ is a non-zero element fo $\mathscr{S}_{k}(M, \chi)$, then $f(w)$ has the Fourier expansion

$$
f(w)=\sum_{n=1}^{\infty} c(n) e^{2 \pi i n w}
$$

at the cusp $\infty$. Hecke proved that the Dirichlet series

$$
L(s)=L(s ; f)=\sum_{n=1}^{\infty} c(n) n^{-s}
$$

is convergent absolutely in the half-plane $\sigma>(k+1) / 2$, and can be continued holomorphically to the whole plane. Furthermore, the functional equation 


$$
\Lambda(s ; f)=i^{k} \Lambda(k-s ; \tilde{f})
$$

is valid, where $\Lambda(s ; f)=(2 \pi / \sqrt{M})^{-s} \Gamma(s) L(s ; f)$ and $\tilde{f}(w)=M^{-k / 2} w^{-k} f(-1 / M w)$. From (2.1) we see that the "critical strip" of $L(s ; f)$ is $\{s \mid(k-1) / 2 \leqq \sigma$ $\leqq(k+1) / 2\}$, and the "critical line" is $\sigma=k / 2$. We consider the valuedistribution of $L(s ; f)$ in the half-plane $\sigma>k / 2$.

Now we assume $f(w)$ is a primitive form of level $M$. Then, $f(w)$ is a simultaneous eigenfunction of Hecke operators $T(n)$, defined by

$$
(f \mid T(n))(w)=n^{k-1} \sum_{\substack{0<d \mid n \\ a d=n}} \sum_{b=0}^{d-1} \chi(a) d^{-k} f((a w+b) / d),
$$

and the corresponding eigenvalue is equal to the $n$-th Fourier coefficient $c(n)$. The Euler product expansion

$$
L(s ; f)=\prod_{n=1}^{\infty}\left(1-c\left(p_{n}\right) p_{n}^{-s}+\chi\left(p_{n}\right) p_{n}^{k-1-2 s}\right)^{-1}
$$

holds for $\sigma>(k+1) / 2$. Hence $L(s) \neq 0$ if $\sigma>(k+1) / 2$, so we can define

$$
\log L(s)=-\sum_{n=1}^{\infty} \log \left(1-c\left(p_{n}\right) p_{n}^{-s}+\chi\left(p_{n}\right) p_{n}^{k-1-2 s}\right)
$$

in this region. Here we comment the rigorous meaning of the right-hand side of the above. If $\left(p_{n}, M\right)=1$, then it follows from Deligne [8] and Deligne-Serre [9] that we can write

$$
1-c\left(p_{n}\right) p_{n}^{-s}+\chi\left(p_{n}\right) p_{n}^{k-1-2 s}=\left(1-\alpha_{n} p_{n}^{-s}\right)\left(1-\beta_{n} p_{n}^{-s}\right)
$$

with $\left|\alpha_{n}\right| \leqq p_{n}^{(k-1) / 2}$ and $\left|\beta_{n}\right| \leqq p_{n}^{(k-1) / 2}$. So the principal value $\log \left(1-\alpha_{n} p_{n}^{-s}\right)$, $\log \left(1-\beta_{n} p_{n}^{-s}\right)$ is well-defined if $\sigma>(k-1) / 2$, and we put

(2.3) $\log \left(1-c\left(p_{n}\right) p_{n}^{-s}+\chi\left(p_{n}\right) p_{n}^{k-1-2 s}\right)=\log \left(1-\alpha_{n} p_{n}^{-s}\right)+\log \left(1-\beta_{n} p_{n}^{-s}\right)$.

Next, if $p_{n} \mid M$, then $\chi\left(p_{n}\right)=0$, and $\left|c\left(p_{n}\right)\right| \leqq p_{n}^{(k-1) / 2}$ since $f(w)$ is primitive. Hence, (2.3) is valid with $\alpha_{n}=c\left(p_{n}\right)$ and $\beta_{n}=0$. Hence, each term in the right-hand side of $(2.2)$ is well-defined for $\sigma>(k-1) / 2$, and the sum is convergent absolutely for $\sigma>(k+1) / 2$.

Next we define $\log L(s)$ in the strip $k / 2<\sigma \leqq(k+1) / 2$. There is a possibility of the existence of zeros of $L(s)$ in this region, so we restrict our consideration to the set

$$
G=\{s \mid \sigma>k / 2\}-\bigcup_{s_{j}=o_{j}+i t_{j}}\left\{s=\sigma+i t_{j} \mid k / 2<\sigma \leqq \sigma_{j}\right\},
$$


where $s_{j}$ 's $(j=1,2, \cdots)$ run through all possible zeros of $L(s)$ in $k / 2<\sigma$ $\leqq(k+1) / 2$. For any $s_{0}=\sigma_{0}+i t_{0} \in G$, we define $\log L\left(s_{0}\right)$ by the analytic continuation along the path $\left\{s=\sigma+i t_{0} \mid \sigma \geqq \sigma_{0}\right\}$.

We fix a $\sigma_{0}>k / 2$, and discuss the value-distribution of $\log L(s)$ on the line $\sigma=\sigma_{0}$. Let $R$ be an arbitrary rectangle, and $T>0$. The set

$$
\left\{t \in[0, T] \mid \sigma_{0}+i t \in G, \log L\left(\sigma_{0}+i t\right) \in R\right\}
$$

consists of several intervals, so it is obviously Jordan measurable, and by $V(T, R)=V(T, R ; L)$ we denote the Jordan measure of this set. The principal result of this paper is the following

Theorem 1. Let $L(s)$ be the Dirichlet series attached to a primitive form of level $M$. Then, there exists the limit

$$
W(R)=W(R ; L)=\lim _{T \rightarrow \infty} V(T, R ; L) / T
$$

for any $\sigma_{0}>k / 2$.

The following four sections are devoted mainly to the proof of Theorem 1. In the proof we shall see that $W$ is a probability measure. The evaluation of $W(E)$ for any measurable $E$ is an interesting problem. In this direction, as a generalization of Theorem 19 of Jessen-Wintner [13], we have

Theorem 2. Let $a, \lambda$ be positive numbers. Then, for any $W$-measurable set $E$ included in $\{z|| z \mid>3 a\}$, the inequality

$$
W(E) \leqq C e^{-\lambda a^{2}}
$$

holds, where $C$ is a positive constant depending only on $\lambda, k$ and $\sigma_{0}$.

\section{§3. Application of the Kronecker-Weyl theorem}

Let $N$ be a positive integer, and put

$$
L_{N}(s)=\prod_{n=1}^{N}\left(1-c\left(p_{n}\right) p_{n}^{-s}+\chi\left(p_{n}\right) p_{n}^{k-1-2 s}\right)^{-1}
$$

Then,

$$
\log L_{N}(s)=-\sum_{n=1}^{N} \log \left(1-c\left(p_{n}\right) p_{n}^{-s}+\chi\left(p_{n}\right) p_{n}^{k-1-2 s}\right),
$$

which is well-defined if $\sigma>(k-1) / 2$. Let $V_{N}(T, R)=V_{N}(T, R ; L)$ be the Jordan measure of the set 


$$
\left\{t \in[0, T] \mid \log L_{N}\left(\sigma_{0}+i t\right) \in R\right\} .
$$

Next, let $Q_{N}=[0,1)^{N}$ be the $N$-dimensional unit torus, and for any $\left(\theta_{1}, \cdots, \theta_{N}\right) \in[0,1)^{N}$, we put

$$
\begin{aligned}
S_{N}\left(\theta_{1}, \cdots, \theta_{N}\right)=-\sum_{n=1}^{N} \log (1-c( & \left.p_{n}\right) p_{n}^{-\sigma_{0}} \exp \left(2 \pi i \theta_{n}\right) \\
& \left.+\chi\left(p_{n}\right) p_{n}^{k-1-2 \sigma_{0}} \exp \left(4 \pi i \theta_{n}\right)\right) .
\end{aligned}
$$

For any subset $E \subset C$, we denote the inverse image $S_{N}^{-1}(E)$ by $\Omega_{N}(E)=$ $\Omega_{N}(E ; L)$. Then, $\log L_{N}\left(\sigma_{0}+i t\right) \in R$ if and only if

$$
\left(\left\{-\left(\frac{\log p_{1}}{2 \pi}\right) t\right\}, \cdots \cdots,\left\{-\left(\frac{\log p_{N}}{2 \pi}\right) t\right\}\right) \in \Omega_{N}(R)
$$

where the symbol $\{x\}$ denotes the fractional part of $x$. Hence, if $\Omega_{N}(R)$ is Jordan measurable, then by using the Kronecker-Weyl theorem (see Titchmarsh [19], §11.7), we can conclude

$$
\lim _{T \rightarrow \infty} V_{N}(T, R ; L) / T=W_{N}(R),
$$

where $W_{N}(R)=W_{N}(R ; L)$ is the $N$-dimensional Jordan measure of $\Omega_{N}(R)$. Therefore, to establish (3.1), it is sufficient to prove the following

Lemma 1. For any rectangle $R$, the set $\Omega_{N}(R)$ is Jordan measurable. Furthermore, for any positive $\varepsilon$, there exists a positive $\eta$, which is independent of $N$, and for which $W_{N}(R)<\varepsilon$ holds for any $R$ with the area $\mu(R)<\eta$.

This lemma was at first proved by Bohr-Courant [2] for the case of $\zeta(s)$, and then, in $\S 11$ of Bohr-Jessen [5] for general convex curves. Their induction argument can be applied to our present case.

Let

$$
z_{n}=z_{n}\left(\theta_{n}\right)=-\log \left(1-c\left(p_{n}\right) p_{n}^{-\sigma_{0}} \exp \left(2 \pi i \theta_{n}\right)+\chi\left(p_{n}\right) p_{n}^{k-1-2 \sigma_{0}} \exp \left(4 \pi i \theta_{n}\right)\right),
$$

and $\omega_{n}=\left\{z_{n}\left(\theta_{n}\right) \mid 0 \leqq \theta_{n}<1\right\}$. We prove the lemma by induction.

The set $\Omega_{1}(R)$ is a union of several intervals, so it is clearly Jordan measurable. To show the second assertion, we first note that if $\mu(R)<\eta$, then the length of at least one edge of $R$ is smaller than $\sqrt{\eta}$, hence it is included in an open strip of width $\sqrt{\eta}$, parallel to the real or imaginary axis. We only treat the former; the argument in the latter case is similar. 
For any real $x$, by an elementary calculation we can show that the number of the roots $\theta_{1}$ which satisfies $\operatorname{Im} z_{1}\left(\theta_{1}\right)=x$ is at most four. Let $l$ be an arbitrary line parallel to the real axis, and denote by $z_{1}\left(\theta_{1}^{(\nu)}\right)$ $(1 \leqq \nu \leqq 4)$ the intersection points of $l$ and $\omega_{1}$. Let $A(l ; \eta)$ be the open strip of width $\eta$, whose center line is $l$. For sufficiently small $\eta$, the strip $A(l ; 2 \sqrt{\eta})$ includes only four disjoint pieces $\omega_{1}^{(\nu)}(l)$ of $\omega_{1}(1 \leqq \nu \leqq 4)$ on which lies the point $z_{1}\left(\theta_{1}^{(\nu)}\right)$, respectively. Furthermore, we can choose $\eta=\eta(l)$ so small that the length of the set $\left\{\theta_{1} \mid z_{1}\left(\theta_{1}\right) \in \omega_{1}^{(\nu)}(l)\right\}$ is less than $\varepsilon / 4$. Hence we have that the Jordan measure of the set

$$
\Omega_{1}(A(l ; 2 \sqrt{\eta(l)}))
$$

is smaller than $\varepsilon$. We define

$$
\omega_{1}(l)=\left(\bigcup_{\nu=1}^{4} \omega_{1}^{(\nu)}(l)\right) \cap A(l ; \sqrt{\eta(l)}) .
$$

Since $\omega_{1}$ is compact, we can choose a finite number of the lines $\left\{l_{j}\right\}$, which gives a finite covering $\left\{\omega_{1}\left(l_{j}\right)\right\}$ of $\omega_{1}$. And we put

$$
\eta=\min _{j}\left\{\eta\left(l_{j}\right)\right\}
$$

Then it is obvious that for any $l$, there exists a line $l_{j}$, for which

$$
\omega_{1} \cap A(l ; \sqrt{\eta}) \subset A\left(l_{j} ; 2 \sqrt{\eta\left(l_{j}\right)}\right)
$$

holds. This implies the second assertion for $N=1$.

The following second step is the same as in the original proof of Bohr-Courant, but we present the argument for the convenience of readers.

We now assume the lemma is valid for $N$. By the assumption, $W_{N}\left(R-z_{N+1}\right)$ is a continuous function of $z_{N+1}$, so is also a continuous function of $\theta_{N+1}$. Hence the integral

$$
I(R)=\int_{0}^{1} W_{N}\left(R-z_{N+1}\right) d \theta_{N+1}
$$

exists.

We denote the four vertices of $R$ by $A_{u}+i B_{v}\left(u, v=1,2, A_{1}<A_{2}\right.$, $\left.B_{1}<B_{2}\right)$ :

$$
R=\left\{z \mid A_{1} \leqq \operatorname{Re}(z) \leqq A_{2}, B_{1} \leqq \operatorname{Im}(z) \leqq B_{2}\right\}
$$

Let $\delta>0$, and we put 


$$
R_{i}=R_{i}(\delta)=\left\{z \mid A_{1}+\delta \leqq \operatorname{Re}(z) \leqq A_{2}-\delta, B_{1}+\delta \leqq \operatorname{Im}(z) \leqq B_{2}-\delta\right\}
$$

and

$$
R_{y}=R_{y}(\delta)=\left\{z \mid A_{1}-\delta \leqq \operatorname{Re}(z) \leqq A_{2}+\delta, B_{1}-\delta \leqq \operatorname{Im}(z) \leqq B_{2}+\delta\right\} .
$$

For any positive $\varepsilon$, by the assumption there exists a sufficiently small $\delta$, independent of $N$, for which the inequalities

$$
\begin{aligned}
& W_{N}\left(R-z_{N+1}\right)-\varepsilon<W_{N}\left(R_{i}-z_{N+1}\right), \\
& W_{N}\left(R_{y}-z_{N+1}\right)<W_{N}\left(R-z_{N+1}\right)+\varepsilon
\end{aligned}
$$

hold for any $z_{N+1} \in \omega_{N+1}$.

Let us take a sequence $0=\theta_{N+1}^{(1)}<\theta_{N+1}^{(2)}<\cdots<\theta_{N+1}^{(m)}<\theta_{N+1}^{(m+1)}=1$, and define

$$
I^{(m)}(R)=\sum_{\mu=1}^{m} W_{N}\left(R-z_{N+1}^{(\mu)}\right)\left(\theta_{N+1}^{(\mu+1)}-\theta_{N+1}^{(\mu)}\right),
$$

where $z_{N+1}^{(\mu)}=z_{N+1}\left(\theta_{N+1}^{(\mu)}\right)$. Under a suitable choice of $\left\{\theta_{N+1}^{(\mu)}\right\}$, we have

$$
\left|I(R)-I^{(m)}(R)\right|<\varepsilon,
$$

and

$$
R_{i}-z_{N+1}^{(\mu)} \subset R-z_{N+1} \subset R_{y}-z_{N+1}^{(\mu)}
$$

for any $\mu$ and any $z_{N+1}=z_{N+1}\left(\theta_{N+1}\right)$ with $\theta_{N+1}^{(\mu)} \leqq \theta_{N+1}<\theta_{N+1}^{(\mu+1)}$. Hence,

$$
\Omega_{N}\left(R_{i}-z_{N+1}^{(\mu)}\right) \times\left[\theta_{N+1}^{(\mu)}, \theta_{N+1}^{(\mu+1)}\right) \subset \Omega_{\mu} \subset \Omega_{N}\left(R_{y}-z_{N+1}^{(\mu)}\right) \times\left[\theta_{N+1}^{(\mu)}, \theta_{N+1}^{(\mu+1)}\right),
$$

where $\Omega_{\mu}=\left\{\left(\theta_{1}, \cdots, \theta_{N+1}\right) \in \Omega_{N+1}(R) \mid \theta_{N+1}^{(\mu)} \leqq \theta_{N+1}<\theta_{N+1}^{(\mu+1)}\right\}$. So it follows that $W_{N}\left(R_{i}-z_{N+1}^{(\mu)}\right)\left(\theta_{N+1}^{(\mu+1)}-\theta_{N+1}^{(\mu)}\right) \leqq \underline{m}\left(\Omega_{\mu}\right) \leqq \bar{m}\left(\Omega_{\mu}\right) \leqq W_{N}\left(R_{y}-z_{N+1}^{(\mu)}\right)\left(\theta_{N+1}^{(\mu+1)}-\theta_{N+1}^{(\mu)}\right)$, where $\underline{m}(X)$ (resp. $\bar{m}(X)$ ) denotes the Jordan inner (resp. outer) volume of $X$, hence the inequality

$$
I^{(m)}\left(R_{i}\right) \leqq \underline{m}\left(\Omega_{N+1}(R)\right) \leqq \bar{m}\left(\Omega_{N+1}(R)\right) \leqq I^{(m)}\left(R_{y}\right)
$$

follows. Combining this result with (3.2), (3.3) and (3.4), we have

$$
I(R)-2 \varepsilon \leqq \underline{m}\left(\Omega_{N+1}(R)\right) \leqq \bar{m}\left(\Omega_{N+1}(R)\right) \leqq I(R)+2 \varepsilon,
$$

which implies $\Omega_{N+1}(R)$ is Jordan measurable, and

$$
W_{N+1}(R)=\int_{0}^{1} W_{N}\left(R-z_{N+1}\right) d \theta_{N+1} .
$$


The second assertion of the lemma is a direct consequence of the expression (3.5).

\section{§4. An evaluation of the probability measure $W_{N}$}

Let $E$ a subset of $C$, for which $\Omega_{N}(E)$ is Lebesgue measurable. We denote the $N$-dimensional Lebesgue measure of $\Omega_{N}(E)$ by $W_{N}(E)$. Then $W_{N}$ is clearly a probability measure over $C$, and, due to Lemma 2.4 .3 of Itô [12], it is regular. The purpose of this section is to prove the following

LEMma 2. Let $\lambda$ be an arbitrary positive number. Then, there exists a positive constant $a_{0}=a_{0}\left(\lambda, k, \sigma_{0}\right)$, for which the inequality

$$
W_{N}(E) \leqq C e^{-2 a^{2}}
$$

holds for any $a>a_{0}$, any Borel set $E \subset\{z|| z \mid>2 a\}$ and any sufficiently large positive integer $N$, with a positive constant $C=C\left(\lambda, k, \sigma_{0}\right)$.

The basic idea of the following proof is due to Jessen-Wintner [13] (see also Borchsenius-Jessen [6]), though their argument depends on the existence of the density function of $W_{N}$.

Let $r$ be a positive integer, $N>r$, and put

$$
\begin{aligned}
S_{r, N}\left(\theta_{r+1}, \cdots, \theta_{N}\right)=-\sum_{n=r+1}^{N} \log (1- & c\left(p_{n}\right) p_{n}^{-\sigma_{0}} \exp \left(2 \pi i \theta_{n}\right) \\
& \left.+\chi\left(p_{n}\right) p_{n}^{k-1-2 \sigma_{0}} \exp \left(4 \pi i \theta_{n}\right)\right) .
\end{aligned}
$$

For any Borel set $E$, the inverse image $\Omega_{r, N}(E)=S_{r, N}^{-1}(E)$ is Lebesbue measurable, so we can define a probability measure $W_{r, N}(E)$, which is equal to the $(N-r)$-dimensional Lebesgue measure of $\Omega_{r, N}(E)$. By Fubini's theorem we have

$$
\begin{aligned}
W_{N}(E) & =\int_{Q_{N-r}} W_{r}\left(E-S_{r, N}\left(\theta_{r+1}, \cdots, \theta_{N}\right)\right) d m\left(\theta_{r+1}, \cdots, \theta_{N}\right) \\
& =\int_{C} W_{r}(E-z) d W_{r, N}(z)
\end{aligned}
$$

where $m$ is the $(N-r)$-dimensional Lebesgue measure.

The set

$$
\sum_{r}=\left\{S_{r}\left(\theta_{1}, \cdots, \theta_{r}\right) \mid \theta_{n} \in[0,1)(1 \leqq n \leqq r)\right\}
$$

is bounded; there exists a positive number $a_{0}=a_{0}\left(r, k, \sigma_{0}\right)$ for which $\sum_{r} \subset$ $\left\{z|| z \mid \leqq a_{0}\right\}$ holds. Let $a>a_{0}$ and $E$ an arbitrary Borel set included in 
$\{z|| z \mid>2 a\}$. If $|z| \leqq a$, then $(E-z) \cap \sum_{r}=\varnothing$, which yields $W_{r}(E-z)$ $=0$. Therefore, from (4.1), we have

$$
\begin{aligned}
W_{N}(E) & =\int_{|z|>a} W_{r}(E-z) d W_{r, N}(z) \\
& \leqq \int_{|z|>a} d W_{r, N}(z)=W_{r, N}(\{z|| z \mid>a\}) .
\end{aligned}
$$

To evaluate the right-hand side of the above, we prepare the following

Lemma 3. Let $\lambda>0, b>0$, and $B$ a bounded set which satisfies $B \subset\{z|| z \mid \leqq b\}$. Then, under a suitable choice of $r=r\left(\lambda, k, \sigma_{0}\right)$, there exists a positive constant $C_{1}=C_{1}\left(\lambda, k, \sigma_{0}\right)$, for which

$$
W_{r, N}\left(z_{0}-B\right) \leqq C_{1} \exp \left(-4 \lambda\left|z_{0}\right|^{2}\right)
$$

holds for any $z_{0} \in\{z|| z \mid>2 b\}$.

Proof. At first we note that if $\theta=\left(\theta_{r+1}, \cdots, \theta_{N}\right) \in \Omega_{r, N}\left(z_{0}-B\right)$, then $\left|S_{r, N}(\theta)\right|>\left|z_{0}\right| / 2$. Hence,

$$
\begin{aligned}
\exp \left(4 \lambda\left|z_{0}\right|^{2}\right) W_{r, N}\left(z_{0}-B\right) & =\int_{\Omega_{r, N}\left(z_{0}-B\right)} \exp \left(4 \lambda\left|z_{0}\right|^{2}\right) d m(\theta) \\
& \leqq \int_{\Omega_{r, N}\left(z_{0}-B\right)} \exp \left(16 \lambda\left|S_{r, N}(\theta)\right|^{2}\right) d m(\theta) \\
& \leqq \int_{Q_{N-r}} \exp \left(16 \lambda\left|S_{r, N}(\theta)\right|^{2}\right) d m(\theta)
\end{aligned}
$$

Next, since $\sigma_{0}>k / 2$, we have

$$
\left|\alpha_{n} p_{n}^{-\sigma_{0}} \exp \left(2 \pi i \theta_{n}\right)\right| \leqq p_{n}^{(k-1) / 2-\sigma_{0}} \leqq 2^{(k-1) / 2-\sigma_{0}}<1 / \sqrt{2}<1,
$$

and the same estimate holds for $\beta_{n} p_{n}^{-\sigma_{0}} \exp \left(2 \pi i \theta_{n}\right)$. There is an absolute constant $C_{2}$, for which

$$
|-\log (1-z)-z| \leqq C_{2}|z|^{2}
$$

holds for any $z \in\{|z| \leqq 1 / \sqrt{2}\}$. Hence, if we put

$$
S_{r, N}^{*}(\theta)=\sum_{n=r+1}^{N}\left(\alpha_{n}+\beta_{n}\right) p_{n}^{-\sigma_{0}} \exp \left(2 \pi i \theta_{n}\right)
$$

then

$$
\left|S_{r, N}(\theta)-S_{r, N}^{*}(\theta)\right| \leqq C_{2} \sum_{n=r+1}^{N}\left(\left|\alpha_{n}\right|^{2}+\left|\beta_{n}\right|^{2}\right) p_{n}^{-2 \sigma_{0}} \leqq 2 C_{2} C_{3}
$$


where

$$
C_{3}=C_{3}\left(k, \sigma_{0}\right)=\sum_{n=1}^{\infty} p_{n}^{k-1-2 \sigma_{0}} .
$$

In general, if $|u-v| \leqq w$, then $|u|^{2} \leqq 2\left(|v|^{2}+w^{2}\right)$. Therefore,

$$
\begin{aligned}
\int_{Q_{N-r}} \exp & \left(16 \lambda\left|S_{r, N}(\theta)\right|^{2}\right) d m(\theta) \\
& \leqq \int_{Q_{N-r}} \exp \left(32 \lambda\left(\left|S_{r, N}^{*}(\theta)\right|^{2}+4 C_{2}^{2} C_{3}^{2}\right)\right) d m(\theta) \\
& =\exp \left(128 C_{2}^{2} C_{3}^{2} \lambda\right) \sum_{j=0}^{\infty} \frac{(32 \lambda)^{j}}{j !} \int_{Q_{N-r}}\left|S_{r, N}^{*}(\theta)\right|^{2 j} d m(\theta) .
\end{aligned}
$$

By using Parseval's equation, we have

$$
\begin{aligned}
\int_{Q_{N-r}}\left|S_{r, N}^{*}(\theta)\right|^{2 j} d m(\theta) & =\sum_{j_{r+1} \cdots+j_{N}=j}\left|\frac{j !}{j_{r+1} ! \cdots j_{N} !} \prod_{n=r+1}^{N}\left(\left(\alpha_{n}+\beta_{n}\right) p_{n}^{-\sigma_{0}}\right)^{j_{n}}\right|^{2} \\
& \leqq j !\left(\sum_{n=r+1}^{N}\left|\left(\alpha_{n}+\beta_{n}\right) p_{n}^{-\sigma_{0}}\right|^{2}\right)^{j}
\end{aligned}
$$

Now we choose $r=r\left(\lambda, k, \sigma_{0}\right)$ so large that

$$
d=1-32 \lambda \sum_{n=r+1}^{\infty}\left|\left(\alpha_{n}+\beta_{n}\right) p_{n}^{-\sigma_{0}}\right|^{2} \geqq 1 / 2
$$

holds. Then we have

$$
\begin{aligned}
& \int_{Q_{N-r}} \exp \left(16 \lambda\left|S_{r, N}(\theta)\right|^{2}\right) d m(\theta) \\
& \quad \leqq \exp \left(128 C_{2}^{2} C_{3}^{2} \lambda\right) \sum_{j=0}^{\infty}(1-d)^{j} \leqq 2 \cdot \exp \left(128 C_{2}^{2} C_{3}^{2} \lambda\right) .
\end{aligned}
$$

This inequality with (4.3) leads to the assertion of Lemma 3.

Now we complete the proof of Lemma 2. Let

$$
\begin{aligned}
& \Delta=\Delta(\mu, \nu)=\left\{z \mid \mu\left(a_{0} / 2 \sqrt{2}\right) \leqq\right. \operatorname{Re}(z) \leqq(\mu+1)\left(a_{0} / 2 \sqrt{2}\right), \\
&\left.\nu\left(a_{0} / 2 \sqrt{2}\right) \leqq \operatorname{Im}(z) \leqq(\nu+1)\left(a_{0} / 2 \sqrt{2}\right)\right\}
\end{aligned}
$$

for any integers $\mu$ and $\nu$. Then it is obvious that

$$
W_{r, N}(\{\boldsymbol{z}|| \boldsymbol{z} \mid>a\}) \leqq \sum_{\Delta} W_{r, N}(\Delta),
$$

where the sum runs through all $\Delta$ which satisfies the condition $\Delta \cap$ $\{z|| z \mid>a\} \neq \varnothing$. Let $z_{\Delta}$ be the vertex of $\Delta$ which is the most distant from the origin. Then we can write $\Delta=z_{\Delta}-\Delta_{0}$, where $\Delta_{0}$ is one of the squares $\Delta(-1,-1), \Delta(-1,0), \Delta(0,-1)$ and $\Delta(0,0)$. Since $\left|z_{\Delta}\right|>a$ and 


$$
\Delta_{0} \subset\left\{z|| z \mid \leqq \sqrt{2}\left(a_{0} / 2 \sqrt{2}\right)=a_{0} / 2\right\},
$$

we can apply Lemma 3 with $z_{0}=z_{\Delta}, B=\Delta_{0}$ and $b=a_{0} / 2$. The result is that

$$
W_{r, N}(\Delta) \leqq C_{1} \exp \left(-4 \lambda\left|z_{\Delta}\right|^{2}\right)
$$

The inequality $|z| \leqq\left|z_{\Delta}\right|$ holds for any $z \in \Delta$, so we have

$$
\exp \left(-4 \lambda\left|z_{\Delta}\right|^{2}\right) \leqq\left(a_{0} / 2 \sqrt{2}\right)^{-2} \int_{\Delta} \exp \left(-4 \lambda|z|^{2}\right) d z
$$

Substituting these results in (4.4), we have

$$
\begin{aligned}
W_{r, N}(\{z|| z \mid>a\}) & \leqq 8 C_{1} a_{0}^{-2} \int_{|z| \geqq a / 2} \exp \left(-4 \lambda|z|^{2}\right) d z \\
& =\left(2 C_{1} \pi / \lambda a_{0}^{2}\right) \exp \left(-\lambda a^{2}\right) .
\end{aligned}
$$

The result of the lemma follows from this inequality and (4.2).

\section{§5. The existence of the asymptotic probability measure}

Borchsenius-Jessen's proof [6] of the existence of $\lim W_{N}(R ; \zeta)$ is based on Lévy's convergence theorem, and their argument can be generalized to our present case. However, by using the result of Lemma 2, we can give a very simple proof of this fact.

Let $P_{1}, P_{2}$ be two regular probability measures over $C$, and $\varepsilon_{12}$ be the lower bound of those $\varepsilon$, for which

$$
P_{1}(F)<P_{2}(\{z \mid \operatorname{dist}(z, F)<\varepsilon\})+\varepsilon
$$

holds for any closed subset $F$. Similarly we define the number $\varepsilon_{21}$, and put

$$
\rho\left(P_{1}, P_{2}\right)=\max \left\{\varepsilon_{12}, \varepsilon_{21}\right\}
$$

It can be shown that $\rho$ is a distance function, which we call Prokhorov's distance. Prokhorov [16] proved that with this metric, the space $\mathscr{D}$ of all regular probability measures over $C$ is a complete separable metric space. The convergence with respect to this metric is equivalent to the weak convergence.

Let $\left\{P_{\alpha}\right\}_{\alpha \in \Lambda}$ be a subset of $\mathscr{D}$. We call $\left\{P_{\alpha}\right\}$ is tight if for any positive $\varepsilon$, there exists a compact set $K=K(\varepsilon) \subset C$, for which the inequality

$$
P_{\alpha}(C-K)<\varepsilon
$$


holds for any $\alpha \in \Lambda$. Now we quote the following

Lemma 4 (Prokhorov [16]). In order for $\left\{P_{a}\right\}$ to be tight it is necessary and sufficient that $\left\{P_{\alpha}\right\}$ is totally bounded with respect to the Prokhorov metric.

If $\sigma_{0}>(k+1) / 2$, then $S_{N}\left(\theta_{1}, \cdots, \theta_{N}\right)$ is uniformly bounded for any $N$, so it is obvious that $\left\{W_{N}\right\}$ is a tight subset. Lemma 2 implies that the tightness is valid for any $\sigma_{0}>k / 2$. Hence, from Lemma 4 , there exists a subsequence $\left\{W_{N(j)}\right\}_{j=1}^{\infty}$, which is convergent weakly to a measure $W \in \mathscr{D}$. In the next section we will prove that this $W$ is just the desired limit in Theorem 1.

Here we note that Theorem 2 is now a immediate consequence of Lemma 2 and the above claim. In fact, let $a>a_{0}$, and $E$ be an $W$ measurable set included in $\{z|| z \mid>3 a\}$. We can assume $E$ is compact, because $W$ is $K$-regular. Let $G_{E}$ be an open set which satisfies

$$
E \subset G_{E} \subset\{z|| z \mid>2 a\} .
$$

Then, there exists a continuous function $g_{E}$ which is equal to 1 on $E$, equal to 0 on $G_{E}^{c}$, and satisfies $0 \leqq g_{E}(z) \leqq 1$ if $z \in G_{E}-E$. Then it follows that

$$
W(E) \leqq \int_{C} g_{E}(z) d W(z)=\lim _{j \rightarrow \infty} \int_{C} g_{E}(z) d W_{N(j)}(z) \leqq \liminf _{j \rightarrow \infty} W_{N(j)}\left(G_{E}\right) .
$$

Lemma 2 shows $W_{N(j)}\left(G_{E}\right) \leqq C e^{-2 a^{2}}$, hence $W(E) \leqq C e^{-\lambda a^{2}}$. To verify Theorem 2 in case $a \leqq a_{0}$, it is enough to change the value of $C$, if necessary.

\section{§ 6. Completion of the proof of Theorem 1}

Let $\varepsilon$ be an arbitrary positive number. The second assertion of Lemma 1 (and its proof) implies that there exists a $\delta>0$, for which

$$
W_{N}\left(R_{y}(2 \delta)-R_{i}(2 \delta)\right)<\varepsilon / 2
$$

holds for any rectangle $R$ and any $N$. We define a continuous function $g_{R}$ by

$$
g_{R}(z)= \begin{cases}1 & \text { if } z \text { is included in the closure of } R_{y}(\delta)-R_{i}(\delta), \\ 0 & \text { if } z \text { is not included in the open kernel of } \\ R_{y}(2 \delta)-R_{i}(2 \delta),\end{cases}
$$

and $0 \leqq g_{R}(z) \leqq 1$ if $z \in\left(R_{i}(\delta)-R_{i}(2 \delta)\right) \cup\left(R_{y}(2 \delta)-R_{y}(\delta)\right)$. Then, 


$$
\begin{aligned}
W\left(R_{y}(\delta)-R_{i}(\delta)\right) & \leqq \int_{C} g_{R}(z) d W(z)=\lim _{j \rightarrow \infty} \int_{C} g_{R}(z) d W_{N(j)}(z) \\
& \leqq \liminf _{j \rightarrow \infty} W_{N(j)}\left(R_{y}(2 \delta)-R_{i}(2 \delta)\right)
\end{aligned}
$$

which yields

$$
\left|W(R)-W\left(R_{i}(\delta)\right)\right|<\varepsilon / 2, \quad\left|W(R)-W\left(R_{y}(\delta)\right)\right|<\varepsilon / 2 .
$$

In particular, any rectangle is a continuity set with respect to $W$. Hence, there exists a sufficiently large positive $J_{1}$, for which

$$
\left|W_{N(j)}\left(R_{i}\right)-W\left(R_{i}\right)\right|<\varepsilon / 2, \quad\left|W_{N(j)}\left(R_{y}\right)-W\left(R_{y}\right)\right|<\varepsilon / 2
$$

holds for any $j \geqq J_{1}$.

Now we assume $\sigma_{0}>(k+1) / 2$. Then we have

$$
\left|\log L\left(\sigma_{0}+i t\right)-\log L_{N(j)}\left(\sigma_{0}+i t\right)\right| \leqq C_{4} \sum_{n=N(j)+1}^{\infty}\left(\left|\alpha_{n} p_{n}^{-s}\right|+\left|\beta_{n} p_{n}^{-s}\right|\right)<\delta
$$

for any real $t$ and any $j \geqq J_{2}$, with a sufficiently large $J_{2}=J_{2}\left(\delta, k, \sigma_{0}\right)$ and an absolute constant $C_{4}$. Hence,

$$
V_{N(j)}\left(T, R_{i}(\delta)\right) \leqq V(T, R) \leqq V_{N(j)}\left(T, R_{y}(\delta)\right),
$$

and so, from (3.1), we have

$$
W_{N(j)}\left(R_{i}\right) \leqq \liminf _{T \rightarrow \infty} V(T, R) / T \leqq \limsup _{T \rightarrow \infty} V(T, R) / T \leqq W_{N(j)}\left(R_{y}\right) .
$$

Hence, with (6.1) and (6.2),

$$
W(R)-\varepsilon \leqq \liminf _{T \rightarrow \infty} V(T, R) / T \leqq \limsup _{T \rightarrow \infty} V(T, R) / T \leqq W(R)+\varepsilon,
$$

which leads to the assertion of Theorem 1 in the domain of absolute convergence.

Next we proceed to the case $k / 2<\sigma_{0} \leqq(k+1) / 2$. By $k_{N}^{i}(T)$ we denote the measure of the set

$$
K_{N}^{\delta}(T)=\left\{t \in[0, T]\left|\sigma_{0}+i t \in G,\right| \log L\left(\sigma_{0}+i t\right)-\log L_{N}\left(\sigma_{0}+i t\right) \mid \geqq \delta\right\} .
$$

Then it follows that

$$
V_{N(j)}\left(T, R_{i}(\delta)\right)-k_{N(j)}^{\delta}(T) \leqq V(T, R) \leqq V_{N(j)}\left(T, R_{y}(\delta)\right)+k_{N(j)}^{\delta}(T)
$$

for any $j$. Let $t_{0}$ be a real number, $k / 2<\alpha_{0}<\sigma_{0}$,

$$
H\left(t_{0}\right)=\left\{s=\sigma+i t \mid \sigma>\alpha_{0}, t_{0}-\frac{1}{2}<t<t_{0}+\frac{1}{2}\right\}
$$


and

$$
\varphi_{N}^{\delta}\left(t_{0}\right)= \begin{cases}0 & \text { if } H\left(t_{0}\right) \subset G, \text { and if }\left|\log L(s)-\log L_{N}(s)\right|<\delta \\ 1 & \text { otherwise. }\end{cases}
$$

Then it is obvious that

$$
k_{N}^{\grave{\delta}}(T) \leqq \int_{0}^{T} \varphi_{N}^{\grave{\delta}}\left(t_{0}\right) d t_{0} .
$$

Hence we have

$$
\begin{aligned}
W_{N(j)}\left(R_{i}\right)-\Phi_{N(j)} & \leqq \liminf _{T \rightarrow \infty} V(T, R) / T \\
& \leqq \limsup _{T \rightarrow \infty} V(T, R) / T \leqq W_{N(j)}\left(R_{y}\right)+\Phi_{N(j)}
\end{aligned}
$$

from (6.4), where

$$
\Phi_{N}=\limsup _{T \rightarrow \infty} T^{-1} \int_{0}^{T} \varphi_{N}^{\delta}\left(t_{0}\right) d t_{0} .
$$

Therefore, if we can show

$$
\lim _{N \rightarrow \infty} \Phi_{N}=0,
$$

then, by a way similar to the case of $\sigma_{0}>(k+1) / 2$, we can complete the proof of Theorem 1 in the critical strip.

In the case of the Riemann zeta-function, the result corresponding to (6.5) is Hilfssatz 5 of Bohr [1]. Bohr's proof of Hilfssatz 5 is based on Hilfssatz 2 in the same paper. The analogue of Hilfssatz 2 in our case can be stated as follows:

LEMMA 5. Let $k / 2<\sigma_{1}<\sigma_{2}$, and $\varepsilon$ be an arbitrary positive number. Then there exists a positive $N_{0}=N_{\circ}\left(\sigma_{1}, \sigma_{2}, \varepsilon\right)$, for which the inequality

$$
\iint_{\substack{\sigma_{1} \leq \sigma \leq \sigma_{2} \\ 0 \leqq t \leqq T}}\left|L(s) / L_{N}(s)-1\right|^{2} d \sigma d t<\varepsilon T
$$

holds for any $N \geqq N_{0}$ and any $T \geqq T_{0}$, with a positive $T_{0}=T_{0}(N)$.

As we have already mentioned in [14], we can skip Bohr's technical argument in the proof of Hilfssatz 2, by using a general mean-value theorem of Carlson.

By virtue of Hecke's estimate (Satz 7 of [11]), we can apply Potter's general result (Theorem 3 of [15]) to our case, and the result is the 
asymptotic formula

$$
\int_{0}^{T}\left|L\left(\sigma_{0}+i t\right)\right|^{2} d t=T \sum_{n=1}^{\infty}|c(n)|^{2} n^{-2 \sigma_{0}}+o(T)
$$

which is valid for $\sigma_{0}>k / 2$. It can be easily shown that

$$
\left|L_{N}\left(\sigma_{0}+i t\right)\right|^{-1} \leqq \exp \left(C_{4} N^{(k+1) / 2-\sigma_{0}}\right),
$$

so from (6.6) we see

$$
T^{-1} \int_{0}^{T}\left|L\left(\sigma_{0}+i t\right) / L_{N}\left(\sigma_{0}+i t\right)-1\right|^{2} d t
$$

is also bounded. Hence, by using Carlson's theorem [7] (see also $\S 9.51$ of Titchmarsh [18]), we have

$$
\lim _{T \rightarrow \infty} T^{-1} \int_{0}^{T}\left|L\left(\sigma_{0}+i t\right) / L_{N}\left(\sigma_{0}+i t\right)-1\right|^{2} d t=\sum_{\substack{\left(m, p_{1} p_{2} \cdots p_{j}\right)=1 \\ m \neq 1}}|c(m)|^{2} m^{-2 \sigma_{0}}
$$

for any $\sigma_{0}>k / 2$, because the Dirichlet series expansion

$$
L(s) / L_{N^{\prime}}(s)=\sum_{\left(m, p_{1} p_{2} \cdots p_{N}\right)=1} c(m) m^{-s}
$$

holds. From the well-known result

$$
\sum_{m \leqq x}|c(m)|^{2}=C_{5} x^{k}+O\left(x^{k-2 / 5}\right)
$$

( $C_{5}$ being a constant depending on $k, M$ and $f$ ) in Rankin's classical work [17], it follows immediately that the right-hand side of (6.7) can be estimated by $O\left(N^{k-2 \sigma_{0}}\right)$ (cf. Lemma 5 of Good [10]). This completes the proof of the lemma.

The method of the deduction of (6.5) from Lemma 5 is quite the same as the original proof of Bohr [1], so we omit the details. Consequently, our Theorem 1 is now proved.

Note added in proof.

The results in the present paper are now generalized to the case of more general Euler products. A generalization of Theorem 1, with a simplified proof, is written in the author's paper entitled "Valuedistribution of zeta-functions", which will be published in "The Proceedings for the Japanese-French Symposium on Analytic Number Theory", ed. by E. Fouvry and K. Nagasaka, a volume in Lecture Notes in Math. Ser., Springer-Verlag. 


\section{REFERENCES}

[1] H. Bohr, Zur Theorie der Riemann'schen Zetafunktion im kritischen Streifen, Acta Math., 40 (1915), 67-100.

[2] H. Bohr and R. Courant, Neue Anwendungen der Theorie der Diophantischen Approximationen auf die Riemannsche Zetafunktion, J. Reine Angew. Math., 144 (1914), 249-274.

[ 3 ] H. Bohr and B. Jessen, Über die Wertverteilung der Riemannschen Zetafunktion, Erste Mitteilung, Acta Math., 54 (1930), 1-35.

[4] ——, Z Z Z - Zweite Mitteilung, ibid., 58 (1932), 1-55.

[5] — - Om Sandsynlighedsfordelinger ved Addition af konvekse Kurver, Dan. Vid. Selsk. Skr. Nat. Math. Afd., (8) 12 (1929), 1-82. = Collected Mathematical Works of H. Bohr, vol. III, 325-406.

[6] V. Borchsenius and B. Jessen, Mean motions and values of the Riemann zeta function, Acta Math., 80 (1948), 97-166.

[ 7 ] F. Carlson, Contributions à la théorie des séries de Dirichlet, Note I, Arkiv för Mat. Astr. och Fysik 16, no. 18 (1922), 19 pp.

[8] P. Deligne, La conjecture de Weil I, Publ. Math. IHES, 43 (1974), 273-307.

[9] P. Deligne and J.-P. Serre, Formes modulaires de poids 1, Ann. Sci. École Norm. Sup. (4) 7 (1974), 507-530.

[10] A. Good, Approximative Funktionalgleichungen und Mittelwertsätze für Dirichletreihen, die Spitzformen assoziiert sind, Comment. Math. Helv., 50 (1975), 327-361.

[11] E. Hecke, Über Modulfunktionen und die Dirichletschen Reihen mit Eulerscher Produktentwicklung I, Math. Ann., 114 (1937), 1-28.

[12] K. Itô, Introduction to probability theory, Cambridge Univ. Press 1984.

[13] B. Jessen and A. Wintner, Distribution functions and the Riemann zeta function, Trans. Amer. Math. Soc., 38 (1935), 48-88.

[14] K. Matsumoto, Discrepancy estimates for the value-distribution of the Riemann zeta-function III, Acta Arith., 50 (1988), 315-337.

[15] H. S. A. Potter, The mean values of certain Dirichlet series I, Proc. London Math. Soc., 46 (1940), 467-478.

[16] Yu. V. Prokhorov, Convergence of random processes and limit theorems in probability theory, Teor. Veroyatnost. i Primenen., 1 (1956), 177-238. = Theory of Probab. Appl., 1 (1956), 157-214.

[17] R. A. Rankin, Contributions to the theory of Ramanujan's function $\tau(n)$ and similar arithmetical functions II, Proc. Cambridge Phil. Soc., 35 (1939), 357-372.

[18] E. C. Titchmarsh, The theory of functions, 2nd ed., Oxford 1939.

[19] - The theory of the Riemann zeta-function, Oxford 1951.

Department of Mathematics

Faculty of Education

Iwate University

Ueda, Morioka 020

Japan 